\title{
Phylogenetic distance in Great Salt Lake microbial communities
}

\author{
J. Jacob Parnell ${ }^{1, *}$, Giovanni Rompato ${ }^{1}$, Todd A. Crowl ${ }^{2,5}$, Bart C. Weimer ${ }^{3}$, \\ Michael E. Pfrender ${ }^{4}$ \\ ${ }^{1}$ Center for Integrated BioSystems and Department of Biology, and ${ }^{2}$ Department of Watershed Sciences and \\ Ecology Center, Utah State University, Logan, Utah 84322, USA \\ ${ }^{3}$ School of Veterinary Medicine, Department of Population Health and Reproduction, \\ University of California at Davis, Davis, California 95616, USA \\ ${ }^{4}$ Department of Biological Sciences, University of Notre Dame, Notre Dame, Indiana 46556, USA
}

${ }^{5}$ Present address: National Science Foundation, Division of Environmental Biology, Arlington, Virginia 22230, USA

\begin{abstract}
Investigations of community composition often rely on metrics based on the abundance of taxonomic groups to estimate biodiversity. Although traditional measures of biodiversity, such as richness and evenness, can be used in a comparative fashion to evaluate differences among communities in both temporal and spatial contexts, these measures generally omit a phylogenetic perspective of the evolutionary diversity represented in a community. Using Fast UniFrac, we examined PhyloChip data from 9 microbial communities throughout the Great Salt Lake, Utah, USA, for changes in phylogenetic distance. We found a significant correlation $(p<0.001)$ between the decreased community phylogenetic distance and increased salt concentration. Despite significant differences in composition, communities in locations with a similar salt concentration had a similar phylogenetic distance. This trend was confirmed by analyzing the biodiversity of 89 published microbial communities classified as extreme $(n=20)$ and non-extreme $(n=69)$. Although we found no significant statistical difference in traditional diversity estimates, such as Chao1 and abundance-based coverage estimate (ACE), between environments, the phylogenetic distance within extreme communities is significantly lower than in non-extreme communities. A smaller phylogenetic distance within more extreme communities may imply evolutionary conservatism and specialization.
\end{abstract}

KEY WORDS: Biodiversity $\cdot$ Hypersaline $\cdot$ Extremophile $\cdot$ Phylogenetic distance $\cdot$ Ecology

\section{INTRODUCTION}

Biodiversity is often defined as the variability among living organisms within ecological systems (Harper \& Hawksworth 1995, Magurran 2004) and is generally calculated using traditional indices such as richness and evenness. However, population geneticists have developed methods that characterize the diversity of populations or groups using phylogenetic or taxonomic differences (Faith 1994, Clarke \& Warwick 1998). Novel diversity indices have been introduced that reflect this variability by characterizing the relatedness or distinctness of organisms within a community
(Nixon \& Wheeler 1990, Vane-Wright et al. 1991, Faith 1992, Solow et al. 1993). In addition to being independent of sample size (Price et al. 1999), the advantage of utilizing phylogenetic distance as opposed to standard diversity estimates in microbial communities is that the functional contribution of a community may depend less on species counts and more on the phylogenetic diversity represented (our Fig. 1; Clarke \& Warwick 1998). The introduction of these methods stems from limitations of traditional diversity indices where each organism is counted equivalently despite high phylogenetic divergence (Fig. 1). One potential result of neglecting the phylogenetic difference between communi- 


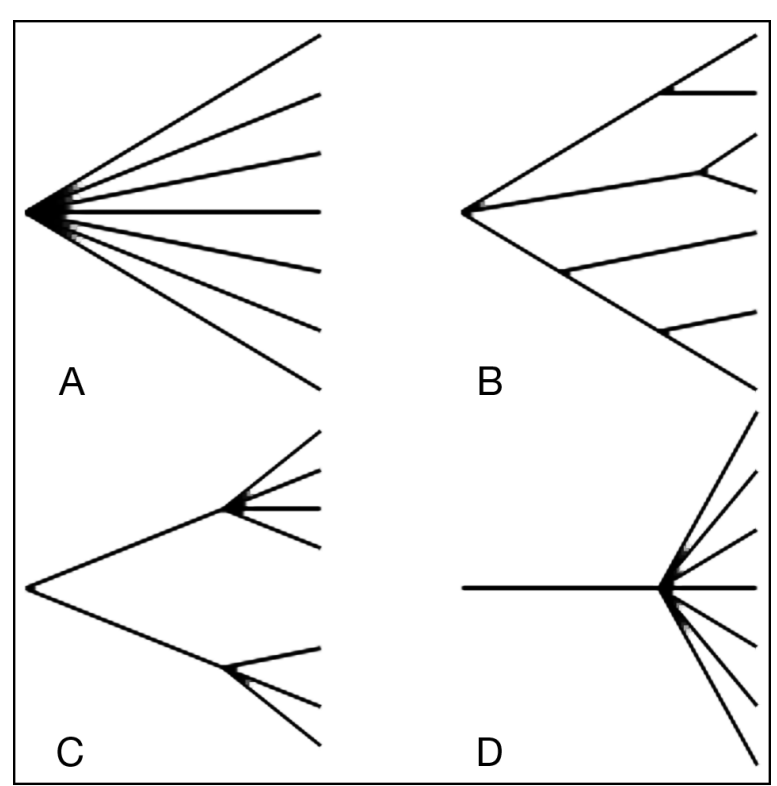

Fig. 1. Representative phylogenetic trees of microbial communities. Despite the same values for richness in each, Community A will most likely have the greatest functional diversity, and functional diversity will decrease $(\mathrm{A}>\mathrm{B}>\mathrm{C}>\mathrm{D})$ as the phylogenetic relatedness increases $(\mathrm{A}<\mathrm{B}<\mathrm{C}<\mathrm{D})$. Figure redrawn from Clarke \& Warwick (2001)

ties is that 2 communities may be considered equally diverse when, in fact, one community is more phylogenetically and functionally diverse than the other (Martin 2002, Hamady et al. 2010). For example, consider the representative communities in Fig. 1. All of the communities have the same number of species, and, at the highest taxonomic resolution (e.g. species or genotype), evenness is also identical. However, the genetic - and consequently functional-difference between these 4 communities is quite distinct. This problem becomes particularly troubling when using biodiversity to infer community function. For example, an over-abundance of closely related groups of species that are functionally redundant (Faith 1994) can lead to a disparity between traditional estimates and functional diversity.

In a previous study, we examined the richness and the taxonomic dispersion between the genus-tospecies ratio and the species-to-genotype-ratio (Parnell et al. 2009). We found a significant loss of genotypic diversity in extreme environments that had experienced disturbances, and we hypothesized that specialization in extreme environments drives the maintenance of genotypic diversity. In the present study we tested this hypothesis by analyzing the phylogenetic distance of 9 microbial communities in the Great Salt Lake, Utah, USA, that differ widely in salinity (Parnell et al. 2010). In addition, we examined 89 published community datasets wherein we asked whether extreme environments (defined by the original authors) harbor more closely related groups than would be expected for non-specialized communities.

\section{MATERIALS AND METHODS}

Case study. We used phylogenetic data from a previous study (Parnell et al. 2010) collected along a salinity gradient, including additional sampling points from the Great Salt Lake (GSL), Utah, USA (Fig. 2). One sample was collected near freshwater inlets into the GSL in Farmington Bay $\left(\mathrm{FB}_{;} 41^{\circ} 03^{\prime} 31.30^{\prime \prime} \mathrm{N}, 112^{\circ} 14^{\prime} 04.98^{\prime \prime} \mathrm{W}\right)$. Three samples were collected from each of 2 sites in the south arm of GSL: Sites A $\left(41^{\circ} 18^{\prime} 48.6^{\prime \prime} \mathrm{N}\right.$, $\left.112^{\circ} 40^{\prime} 59^{\prime \prime} \mathrm{W}\right)$ and B ( $\left.41^{\circ} 07^{\prime} 16.9^{\prime \prime} \mathrm{N}, 112^{\circ} 33^{\prime} 03.5^{\prime \prime} \mathrm{W}\right)$; these samples were taken at the surface (A and $B$ surface), within the water column (A and B column), and and at the bottom (A and B bottom) near the sediments (ca. $3 \mathrm{~m}$ depth). Another surface sample was collected near Antelope Island ( $\mathrm{AI}_{;} 4^{\circ} 02^{\circ} 22.37^{\prime \prime} \mathrm{N}$, $\left.112^{\circ} 16^{\prime} 42.33^{\prime \prime} \mathrm{W}\right)$. One sample was collected from

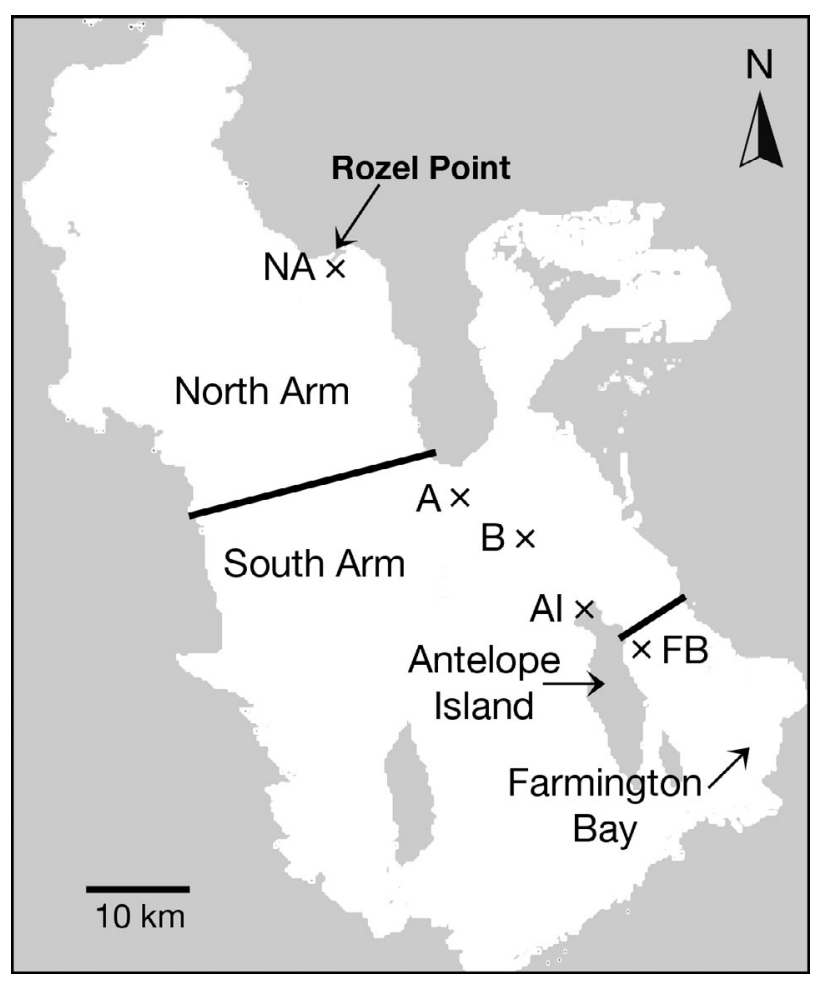

Fig. 2. Sample site locations along a salinity gradient in the Great Salt Lake, Utah, USA. Farmington Bay (FB) is the least saline. South arm samples - AI (near Antelope Island) and the 6 samples taken from Sites A and B-are from locations with intermediate salinity; the samples taken from Sites A and B include depth samples. The north arm (NA) sample, collected near Rozel Point, is near salt saturation. Black lines indicate causeway structures 
the salt-saturated brine of the north arm (NA; $\left.41^{\circ} 25^{\prime} 56.13^{\prime \prime} \mathrm{N}, 112^{\circ} 39^{\prime} 48.31^{\prime \prime} \mathrm{W}\right)$ of GSL near Rozel Point. The least extreme environment was near the freshwater inlet into the lake, where salt concentrations are approximately twice that of marine environments. The microbial community collected from the waters of the southern arm of the lake inhabits an environment with an intermediate $(\sim 15 \%)$ salt concentration. The north end of the lake, the site for collection of the extremophilic hypersaline community, was at salt saturation.

Total DNA was extracted from the hypersaline waters of the GSL as described by Griffiths et al. (2000) using modified hexadecyltrimethylammonium bromide (CTAB) extraction buffer (Zhou et al. 1996). Bead-beating was used to lyse cells, and DNA was extracted with chloroform (Griffiths et al. 2000). The extracted community DNA was purified through a Sephacryl ${ }^{\circledR}$ S-300 column (Parnell et al. 2010).

To assess microbial diversity we used the $16 \mathrm{~S}$ Phylogenetic Array (PhyloChip) that contained probes for 8741 bacterial and archaeal taxa (Brodie et al. 2007). Hybridization of the PhyloChip is achieved using slightly modified Affymetrix protocols. The 16S rRNA was amplified by PCR with Bacteria-specific primers (8F: 5'-AGA GTT TGA TCC TGG CTC AG-3'; 1512R: 5'-ACG GCT ACC TTG TTA CGA CTT-3') or Archaea-specific primers (F: 5'-GAC GGG CGG TGT GTC A-3'; R: 5'-GCG GAT CCG CGG CCG CTG CAG ATC-3') (Parnell et al. 2010). To minimize the primer bias, PCR amplification was performed with a temperature gradient from 48 to $58^{\circ} \mathrm{C}$ for the annealing temperature. The PCR products from the different amplification reactions were collected, purified (QIAquick, Qiagen) and quantified. Fragmentation, labeling, and hybridization were done as mentioned previously (Parnell et al. 2010).

Presence/absence values were determined using probe pair scores by Phylotrac analysis (www.phylotrac.org). Probe pairs scored as positive met 2 criteria: (1) the intensity of fluorescence from the perfect match probe has to be greater than 1.3 times the intensity from the mismatch control; and (2) the difference in intensity (perfect match - mismatch) has to be at least 500 times greater than the squared noise value $(>500$ $N^{2}$; see Brodie et al. 2006). Phylotrac data were imported into Fast UniFrac (http://bmf2.colorado.edu/ fastunifrac) as per Hamady et al. (2010) for community comparisons. Phylogenetic classifications of PhyloChip data were weighted by class, order and family for subsequent community comparisons (Clarke \& Warwick 2001).

Meta analysis. Community biodiversity information was obtained by downloading the 16S rRNA sequence information of 89 randomly selected microbial commu- nities (each containing between 100 and 726 sequences) from the ribosomal database project (http:// rdp.cme.msu.edu) as mentioned previously (Parnell et al. 2009). Microbial communities were from a wide range of globally distributed environmental settings amounting to over 18000 total sequences (see Table S1 in the supplement at www.int-res.com/articles/suppl/ a064p267_supp.pdf). After collecting microbial community datasets, we divided the datasets into 2 categories based on the environmental characteristics of each community as originally defined by the authors (Table 1). Briefly, datasets were categorized as 'extreme' ( $n=20$ ) based on the description of the environments from which the community data were collected (Table S1): environments with high pressure (i.e. deep ocean), extreme temperatures, high salinity, low $\mathrm{pH}$, or environments that were contaminated with solvents; communities with relatively normal environmental parameters ( $\mathrm{n}=69$ ) were termed 'non-extreme'. The average sample size for extreme and non-extreme communities was not significantly different, minimizing sampling issues. We recognize the fact that extreme and non-extreme environments are not discrete, but rather a continuum, and omitted communities whose category would be considered uncertain; some extremophilic environmental details are included in Table 1.

Each microbial community was analyzed with DOTUR (Schloss \& Handelsman 2005) for biodiversity using the Simpson index (Chazdon et al. 1998, Hughes et al. 2001, Magurran 2004), the Shannon evenness index (Magurran 2004), and the abundance-based coverage estimate (ACE) (Chazdon et al. 1998, Hughes et al. 2001, Magurran 2004).

Data on microbial biodiversity, including evenness, richness and phylogenetic distance components, were examined using descriptive and inductive analyses for a difference in extreme environments. In order to normalize residuals, Simpson index data were transformed using the negative natural log (Rosenzweig 1995). Likewise, in order to compensate for hetero-

Table 1. Summary of environmental conditions supporting microbial communities $(n=89)$ analyzed in this study. BOD: biological oxygen demand

\begin{tabular}{|lclc|}
\hline Non-extreme & $\mathrm{n}$ & Extreme & $\mathrm{n}$ \\
\hline Fresh water & 6 & Oligotrophic $(\mathrm{BOD}<1 \mathrm{ppm})$ & 2 \\
Marine water & 11 & Radiation $\left(>50 \mu \mathrm{Ci} \mathrm{g}^{-1}\right)$ & 1 \\
Sediments & 4 & Low pH $(<4.5)$ & 2 \\
Soils & 26 & Low temperature $\left(<5^{\circ} \mathrm{C}\right)$ & 5 \\
Microbiome & 13 & Contaminated & 5 \\
Waste treatment & 9 & Hypersaline $(>7 \%)$ & 3 \\
& & High pressure & 2 \\
& 69 & & 20 \\
\hline
\end{tabular}


scedasticity and to normalize residuals, we used natural log-transformed ACE values. We compared the variance within extreme and non-extreme communities using Student's t-test for independent samples in order to compensate for the different sample sizes. Statistical analyses and graphical output were performed using JMP8 software (SAS).

The Simpson diversity index is a composite value that captures both evenness and richness characteristics of community assemblages (Magurran 2004) and is a robust measure for statistical analyses. In addition, the Simpson diversity index is relatively insensitive to undersampling (Chao \& Shen 2003). Evenness of microbial communities was determined using the Shannon evenness measure as described by Magurran (2004). The ACE was calculated following Hughes et al. (2001) and Magurran (2004). In addition to approximating richness using the $\mathrm{ACE}$, we verified richness patterns using the Chao1 estimate of richness as described by Magurran (2004).

We used a quantitative measure of genetic diversity similar to that using the branch length for the phylogenetic tree (Faith 1992, 1994). Specifically, the genetic distance for each community was determined by the average distance for all members within the community calculated from distance matrix.

\section{RESULTS AND DISCUSSION}

\section{Case study}

In order to determine how the degree of environmental extremity affects phylogenetic distance, we compared 9 microbial communities examined previously along a salinity gradient in the Great Salt Lake, Utah, USA. In this case study, the phylogenetic distance was reached using a qualitative approach (Clarke \& Warwick 2001) due to the qualitative nature of the phylogenetic data (Parnell et al. 2010). The microbial community richness ranged from 1114 identified organisms (Archaea and Bacteria) at the lowest salinity ( $\mathrm{FB}$; approximately $8 \% \mathrm{NaCl}$ ) to 145 organisms at salt saturation (NA).

UniFrac clustering demonstrates the influence of salt concentration on community composition (Fig. 3A); this separation of communities was confirmed using principal coordinate analysis (Fig. 3B). Despite significant differences in individual communities within similar salt concentrations (all south arm samples; intermediate salt) using the Fast UniFrac p-test (corrected $p<0.05$ for all community comparisons except for Abottom vs. Bsurface and Acolumn vs. Bbottom) and the UniFrac significance test (corrected $p<0.05$ for community comparisons: Abottom vs. Bcolumn, Abot-
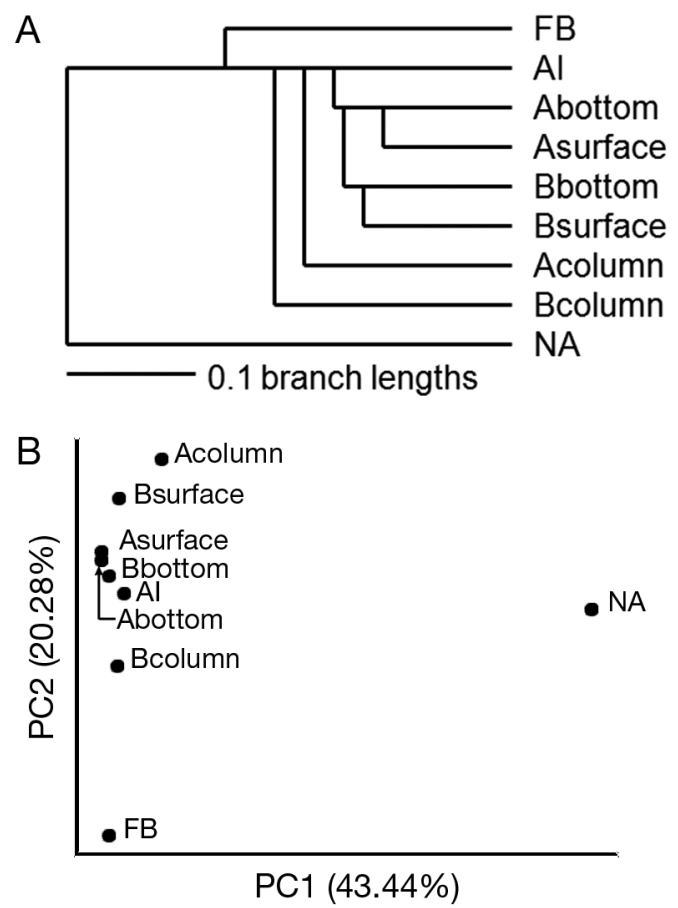

Fig. 3. Statistical grouping of microbial communities from the Great Salt Lake, Utah, USA, using Fast UniFrac. (A) Cluster analysis, and (B) and principal coordinate analysis of microbial communities in increasingly saline environments. The sampling sites A and B are shown in Fig. 2. AI: Antelope Island; FB: Farmington Bay; NA: North Arm. 'column' refers to samples taken within the water column; 'bottom' refers to samples taken near the sediments (ca. $3 \mathrm{~m}$ depth)

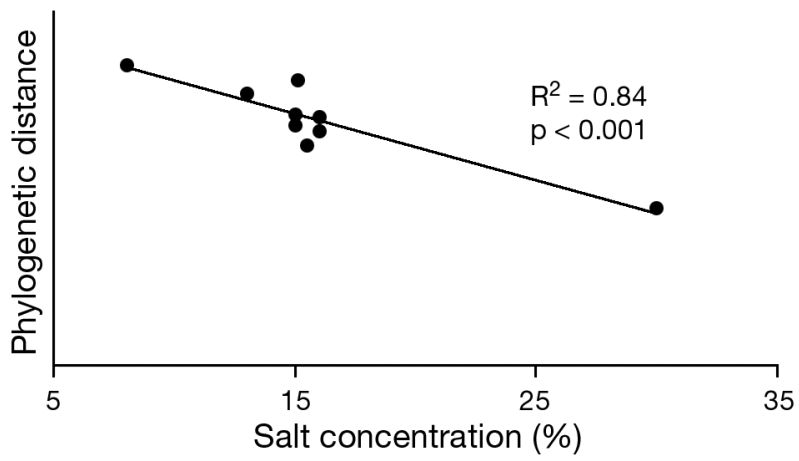

Fig. 4. Correlation between phylogenetic distance of microbial communities and salt concentration of sampling sites throughout the Great Salt Lake

tom vs. Bsurface, Bbottom vs. Bsurface, and Bcolumn vs. Bsurface), the phylogenetic distance within these communities was similar. We found a significant correlation $(p<0.001)$ between higher salinity environments and lower phylogenetic distance (Fig. 4). Difference in potential community function with respect to taxonomic richness is illustrated in archaeal communities throughout the salinity gradient. Archaeal communities in the south arm are represented by both Crenar- 
chaeota and Euryarchaeota with a large number of methanogenic and halophilic groups, respectively. Although the NA sample contained much more archaeal richness than any other sample, all types were within the family Halobacteriaceae (no members of the Crenarchaeota were detected), suggesting evolutionary specialization to extreme conditions.

\section{Meta analysis}

The microbial communities from extreme environments $(n=20)$ had a mean richness estimate of 427 to 484 OTUs, depending on the index used (Chao-ACE). Although this estimate appears to be lower than the richness estimate for non-extreme environments (741 to 817 ), the variability between communities within the same category is high, making this difference not significant (Chao, $\mathrm{p}=0.08$; $\mathrm{ACE}, \mathrm{p}=0.12$ ). Similarly, the Simpson index (ln-transformed) appears lower in extreme environments (4.58 vs. 5.21 in non-extreme environments), but this difference is also not statistically significant ( $p>0.05$ ). Rarefaction curves of the communities analyzed indicate that the sample size effect is minimized, as shown previously (Parnell et al. 2009). It should be noted that this study does not control for the different PCR primers or conditions used in individual cases.

Although traditional estimates did not show significantly less community diversity in extreme environments, compared with non-extreme environments, the phylogenetic distance is significantly lower $(p=0.03)$.
If specialists are significantly clustered phylogenetically, then the mean phylogenetic distance falls lower than the null distribution (Silvertown et al. 2006). Community ecology studies have shown that resource limitations scale positively with phylogenetic similarity due to increased species packing (Tello \& Stevens 2010). Similarly, in extreme environments, where other limitations exist, the phylogenetic distance of these communities suggests a higher tendency toward closely related organisms (Fig. 5). The effect of harsh environmental conditions on phylogenetic diversity indicates that closely related species might have tolerances to similar environmental stressors and thus be more likely to occur within the same community than to occur with less-related species (e.g. Webb 2000).

Both extreme and non-extreme categories fit a normal distribution (Fig. 5) of phylogenetic distance among communities; however, the communities of extreme environments appear to have some multi-modality. By subdividing the extreme categories into groups of temperature, salinity, $\mathrm{pH}$, and contamination, we found that the communities near the mean consisted of contaminated sediments, hypersaline and low-temperature environments, and high-pressure (deep ocean) sediments. The high phylogenetic divergence of these communities may suggest a convergent adaptation to extreme environments (Webb et al. 2002) — that several different phylogenetic groups have evolved different mechanisms to overcome a similar stress. An example of this convergent evolutionary strategy is seen in the adaptation of halophilic organisms to life in high salt concentrations, where at least 2 vastly different
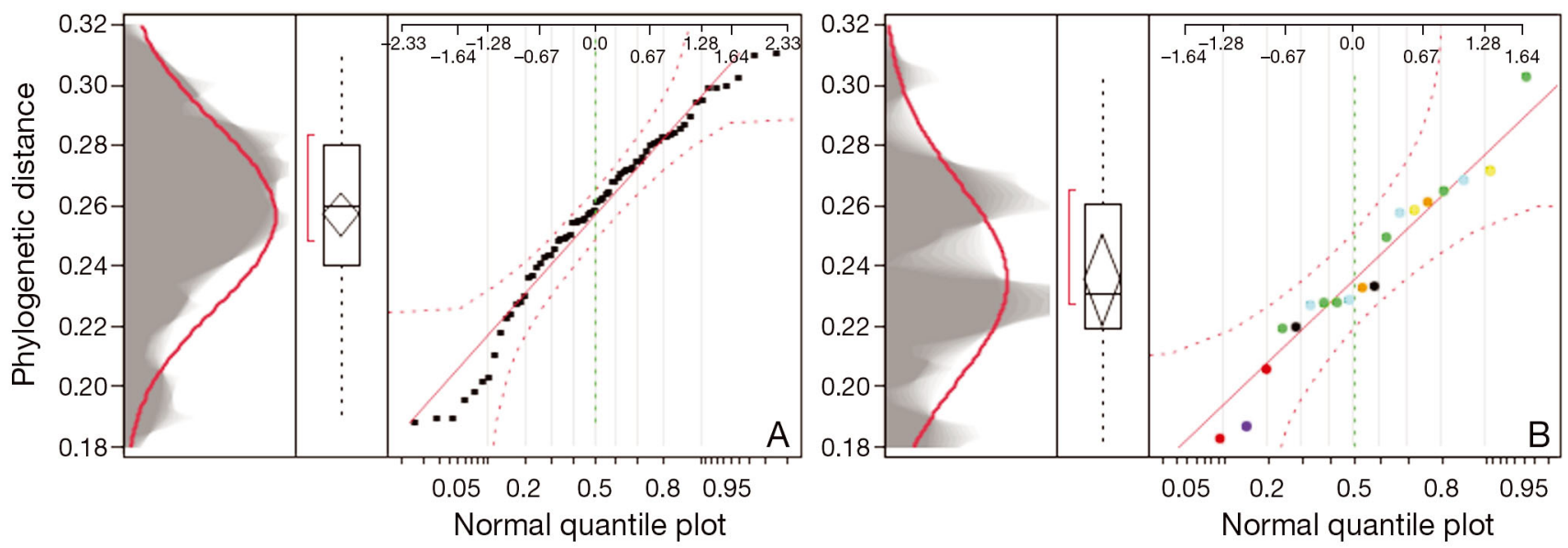

Fig. 5. Distribution of phylogenetic distance between microbial communities in (A) non-extreme and (B) extreme environments. Shadow histograms show the distribution of communities ( $x$-axis) with respect to phylogenetic distance ( $y$-axis). The red line indicates normal distribution; normal quantile plots illustrate how closely data follow normal distribution and suggest that communities in both non-extreme and extreme environments follow a normal distribution. Box-plots illustrate that datasets from nonextreme and extreme environments are not skewed and delineate the upper and lower quartiles, diamonds designate $95 \%$ confidence intervals. Communities in extreme environments are colored as follows: oligotrophic $=$ red; radiation $=$ dark blue; contaminated $=$ green $;$ hypersaline $=$ orange $;$ acidic $=$ black; high pressure $=$ yellow; low temperature $=$ light blue 
mechanisms are involved in regulating osmotic pressure (Oren 2002). Low $\mathrm{pH}$, high radiation, and resource-limited (oligotrophic) environments correspond to higher phylogenetic similarity. In previous studies, this close grouping of phylogeny has suggested that community organization (i.e. the role of competition) can be deduced from the ecological similarity within a closely related group (Webb 2000) and implies habitat selection for ecologically similar, phylogenetically related species (Webb et al. 2002), resulting in a conserved trait within the pool of species in the community. It is unclear whether the type of extreme environment plays a role in the phylogenetic divergence of the community.

\section{CONCLUSION}

Population ecology studies have shown that different organisms make unequal contributions to diversity and ecosystem function due to the amount of variability within genetic or morphological characteristics (May 1990, Humphries et al. 1995, Crozier 1997, Norberg et al. 2001, Allen et al. 2009). Phylogenetic distance (often referred to as phylogenetic diversity or taxonomic diversity) measures the average phylogenetic distance between individual organisms within a community and has been successfully applied to microbial communities, demonstrating a potential to distinguish ecological differences (Martin 2002). As an example, we show that higher archaeal taxonomic richness (at the species level) in the salt-saturated brine of GSL corresponds with specialization rather than with high functional diversity. Communities in the south arm with lower richness have higher phylogenetic distance and greater potential functional diversity.

Understanding microbial biodiversity and its relationship to ecosystem function is a central component of microbial ecology and one of the key questions in science (Huber et al. 2007). In order to address this question we need novel metrics that can link biodiversity with evolutionary history and community structure. Phylogenetic diversity is an important aspect of measuring the total microbial biodiversity of an ecosystem $_{i}$ in the case of the communities examined here, phylogenetic distance is the only significantly different measure between extreme and non-extreme microbial biodiversity. Used in conjunction with traditional biodiversity estimates, phylogenetic diversity is a useful tool for understanding how communities are structured. The work described in the present study is obviously restricted in its coverage and is limited by the datasets examined. Due to the variable nature of the communities examined, a direct comparison of specific phylogenetic groups is not appropriate. However, phylogenetic distance is a well established method of evaluating ecological and evolutionary mechanisms that promote species diversity and co-existence in community ecology (Losos 1996, Webb et al. 2002). In the present study, smaller phylogenetic distance within extreme communities (rather than within non-extreme communities) implies evolutionary conservatism in the specialist group (Silvertown et al. 2006). In this light, a phylogenetic perspective of studying microbial communities provides a new approach to competition and the maintenance of diversity.

Acknowledgements. Funding for this research was provided by a National Science Foundation grant DEB-021212487 to M.E.P., a grant from United States Department of Agriculture CSREES 2006-34526-17001 and support from the Utah Agricultural Experiment Station at Utah State University as journal paper number 8158. T.A.C. was partially supported while serving at the National Science Foundation, Virginia, USA. Any opinion, findings, and conclusions or recommendations expressed in this document are those of the authors and do not necessarily reflect the views of the National Science Foundation.

\section{LITERATURE CITED}

Allen B, Kon M, Bar-Yam Y (2009) A new phylogenetic diversity measure generalizing the Shannon index and its application to phyllostomid bats. Am Nat 174:236-243

Brodie EL, DeSantis TZ, Joyner DC, Baek SM and others (2006) Application of a high-density oligonucleotide microarray approach to study bacterial population dynamics during uranium reduction and reoxidation. Appl Environ Microbiol 72:6288-6298

Brodie EL, DeSantis TZ, Moeberg Parker JJ, Zubietta IX and others (2007) Urban aerosols harbor diverse and dynamic bacterial populations. Proc Natl Acad Sci USA 104: 299-304

Chao A, Shen TJ (2003) Nonparametric estimation of Shannon's index of diversity when there are unseen species in sample. Environ Ecol Stat 10:429-443

Chazdon RL, Colwell RK, Denslow JS, Guariguata MR (1998) Statistical methods for estimating species richness of woody regeneration in primary and secondary rain forests of northeastern Costa Rica. In: Dallmeier F, Comiskey JA (eds) Forest biodiversity research, monitoring and modeling: conceptual background and Old World case studies. Parthenon Publishing, Paris, p 285-309

> Clarke KR, Warwick RM (1998) A taxonomic distinctness index and its statistical properties. J Appl Ecol 35:523-531

Clarke KR, Warwick RM (2001) A further biodiversity index applicable to species lists: variation in taxonomic distinctness. Mar Ecol Prog Ser 216:265-278

> Cole JR, Chai B, Farris RJ, Wang Q and others (2007) The ribosomal database project (RDP-II): introducing myRDP space and quality controlled public data. Nucleic Acids Res 35:D169-D172

> Crozier R (1997) Preserving the information content of species: genetic diversity, phylogeny, and conservation worth. Annu Rev Ecol Evol Syst 28:243-268

- Faith DP (1992) Conservation evaluation and phylogenetic diversity. Biol Conserv 61:1-10 
Faith DP (1994) Phylogenetic diversity: a general framework for the prediction of feature diversity. In: Forey PL, Humphries CJ, Vane-Wright RI (eds) Systematics and conservation evaluation. Systematics Association Special Volume No. 50. Clarendon Press, Oxford, p 251-268

Griffiths RI, Whiteley AS, O'Donnell AG, Bailey MJ (2000) Rapid method for coextraction of DNA and RNA from natural environments for analysis of ribosomal DNA- and rRNA-based microbial community composition. Appl Environ Microbiol 66:5488-5491

> Hamady M, Lozupone C, Knight R (2010) Fast UniFrac: facilitating high-througput phylogenetic analyses of microbial communities including analysis of pyrosequencing and PhyloChip data. ISME J 4:17-27

Harper JL, Hawksworth DL (1995) Biodiversity: measurement and estimation. Chapman \& Hall, London

Huber JA, Welch DBM, Morrison HG, Huse SM, Neal PR, Butterfield DA, Sogin ML (2007) Microbial population structures in the deep marine biosphere. Science 318: 97-100

Hughes JB, Hellmann JJ, Ricketts TH, Bohannan BJM (2001) Counting the uncountable: statistical approaches to estimating microbial diversity. Appl Environ Microbiol 67: 4399-4406

Humphries C, Williams P, Vane-Wright R (1995) Measuring biodiversity value for conservation. Annu Rev Ecol Evol Syst 26:93-111

Losos J (1996) Phylogenetic perspectives on community ecology. Ecology 77:1344-1354

Magurran AE (2004) Measuring biological diversity. Blackwell, Oxford

Martin AP (2002) Phylogenetic approaches for describing and comparing the diversity of microbial communities. Appl Environ Microbiol 68:3673-3682

May R (1990) Taxonomy as destiny. Nature 347:129-130

Nixon KC, Wheeler QD (1990) An amplification of the phylogenetic species concept. Cladistics 6:211-223

Norberg J, Swaney DP, Dushoff J, Lin J, Casagrandi R, Levin SA (2001) Phenotypic diversity and ecosystem functioning in changing environments: a theoretical framework. Proc

Editorial responsibility: Jed Fuhrman,

Los Angeles, California, USA
Natl Acad Sci USA 98:11376-11381

Oren A (2002) Molecular ecology of extremely halophilic Archaea and Bacteria. FEMS Microbiol Ecol 39:1-7

Parnell JJ, Crowl TA, Weimer BC, Pfrender ME (2009) Biodiversity in microbial communities: system scale patterns and mechanisms. Mol Ecol 18:1455-1462

Parnell JJ, Rompato G, Latta LC, Pfrender ME and others (2010) Functional biogeography as evidence of gene transfer in hypersaline microbial communities. PLoS ONE 5: e12919

Price ARG, Keeling MJ, O'Callaghan CJ (1999) Ocean-scale patterns of 'biodiversity' of Atlantic asteroids determined from taxonomic distinctness and other measures. Biol J Linn Soc 66:187-203

Rosenzweig ML (1995) Species diversity in space and time. Cambridge University Press, Cambridge

Schloss PD, Handelsman J (2005) Introducing DOTUR, a computer program for defining operational taxonomic units and estimating species richness. Appl Environ Microbiol 71:1501-1506

Silvertown J, Dodd M, Gowing D, Lawson C, McConway K (2006) Phylogeny and the hierarchical organization of plant diversity. Ecology 87(Suppl):39-49

Solow AR, Polasky S, Broadus JM (1993) On the measurement of biological diversity. J Environ Econ Manag 24:60-68

Tello JS, Stevens RD (2010) Multiple environmental determinants of regional species richness and effects of geographic range size. Ecography 33:796-808

- Vane-Wright RI, Humphries CJ, Williams PH (1991) What to protect?-Systematics and the agony of choice. Biol Conserv 55:235-254

Webb CO (2000) Exploring the phylogenetic structure of ecological communities: an example for rain forest trees. Am Nat 156:145-155

Webb CO, Ackerly DD, McPeek MA, Donoghue MJ (2002) Phylogenies and community ecology. Annu Rev Ecol Evol Syst 33:475-505

Zhou J, Bruns MA, Tiedje JM (1996) DNA recovery from soils of diverse composition. Appl Environ Microbiol 62: $316-322$

Submitted: February 7, 2011; Accepted: June 10, 2011 Proofs received from author(s): September 2, 2011 